a very loose one, contrary to what is observed.

\section{Magnetic Influences}

The presence of magnetic fields on and above the solar surface is a well-established fact. Sunspots are known to possess fields in the kilogauss range, eclipse observations show clearly a shaping of the corona reminiscent of magnetic structures, and magnetic fields are carried along by the solar wind where they are directly measured. Fields at the photospheric level can be inferred from the Zeeman splitting of spectral lines. No direct measurement of corona fields is available so far and does not appear likely in the near future. From the measured photospheric line-of-sight fields, however, it is possible, under given assumptions, to extrapolate a three-dimensional structure of the solar magnetic field at higher levels. These extrapolations present a striking similarity with the emission features observed in the $\mathrm{X}$-ray wavelength range in a number of space experiments and especially during the three Skylab Missions in 1972-73.

These observations proved that the corona is far from being homogeneous and actually presents such dramatic differences from one region to another that it appears to be a collection of widely different entities. The X-ray emission is concentrated in bright structures that have the form of arches (coronal loops) which group together to form the so-called active regions. Contrasting with these bright areas, there are regions of very reduced emission (coronal holes) that are typically found near the solar poles, but may occasionally extend to the equator or even form an elongated dark strip along the meridian connecting one polar region to the other.

All these coronal structures are in a very dynamic state. They appear, evolve, dissapear over a large variety of timescales from minutes to months. From the extrapolations of photospheric fields we see that apart from their intrinsic emissivity, loops and holes differ also for the topology of the associated magnetic fields, coronal holes corresponding to open and coronal loops to closed field structures. There are also clear indications that the solar wind, or at least the high speed streams, flows preferentially from coronal holes.

The extreme structuring of the solar corona suggests that rather than considering the properties of the solar corona on a global scale, i.e. averaging over the individual structures, one should study the properties of the single constituents. This explains why most of the recent work has concentrated on the properties of the coronal loops. They form a subset of the corona for which a large amount of high quality observational material is available and seem to be amenable to a rather detailed theoretical investigation. They are also the seat of coronal flares pictorially described as explosions in a magnetized plasma. The plasma physics of coronal loops poses a variety of exciting and subtle problems.

\section{A Composite View}

Let us however return to the problem of coronal heating and include the magnetic field in our basic parameters. The solar plasma is highly conducting and thus the field lines are tied up with matter. However in the photosphere, the gas pressure exceeds the magnetic pressure (the plasma parameter $\beta>1$ ) whereas the opposite is true in the corona. Turbulent movements at the photospheric levels, induce magnetic stresses that propagate upwards where they can relax, giving up the magnetic energy associated with them. The propaga- ting magnetic stresses can be alternatively described as magnetohydrodynamic waves (instead of the acoustic waves previously considered) or as currents. The study of the transformation of magnetic energy into heat and bulk motion of particles constitutes one of the main objectives of modern coronal physics.

\section{SUGGESTED READING}

1. Vaiana G.S. and Rosner R., "Recent Advances in Coronal Physics", Annual Review of Astronomy and Astrophysics, 16 (1978) 393.

2. Solar Phenomena in Stars and Stellar Systems, Eds. R.M. Bonnet and A.K. Dupree, NATO Advanced Study Institutes Series, (Reidel Publ. Co., Dordrecht) 1981

3. Solar Flare Magnetohydrodynamics, Ed. E.R. Priest, (Gordon and Breach Science Publ., New York) 1981.

\title{
Magnetic Face of the Sun
}

\author{
Cornelis Zwaan, Utrecht \\ (Sterrewacht "Sonnenborgh")
}

The investigation of sunspot physics started with the application of spectroscopy and polarimetry by Hale beginning in 1905 at Mt. Wilson. Hale found that sunspots are much cooler than the ambient solar atmosphere. Furthermore he detected the presence of strong magnetic fields from the Zeeman effect in sunspot spectral lines.

The strong magnetic field is the basic property of sunspots. In the dark inner part, called the umbra, (Fig. 1), the field is nearly vertical; across the surrounding penumbra the field is inclined progressively, becoming nearly horizontal at the outer edge. Thus the field resembles the magnetic field near the end of a long solenoid lying vertically below the sunspot.

The field intensity $B$ measured in the umbral cores is 0.25 to $0.33 \mathrm{~T}$ in large spots, and $0.19<B<0.25 \mathrm{~T}$ in the smallest spots and pores.

There is magnetic flux outside sunspots as well, in discrete elements (faculae) arranged in patterns called facular plages and the network (Fig. 2). In white light these faculae are not conspicuous near the centre of the solar disk, but they stand out as bright features near the solar limb. They are bright all over the disk (Fig. 2c) in the cores of strong spectral lines formed at larger heights.

Since 1966, indications have been accumulated which lead to the conclusion that outside sunspots, the magnetic field also consists of discrete elements of high intensity: $0.10<B<0.20 \mathrm{~T}$, separated by an atmosphere without a measurable magnetic field. The observational proofs did not come easily, as the magnetic elements outside sunspots are quite small: their diame- ters are $400 \mathrm{~km}$ or less, just below the resolution limit of ground-based telescopes during the best observing conditions. Moreover, the "filling factor" of magnetic structure is small: in the photosphere, which is the deep atmosphere where the bulk of the optical radiation originates, the magnetic structure occupies at most $20 \%$ in facular plages in the vicinity of sunspots (see Fig. 2), and much less than $1 \%$ in the "quiet" photosphere.

Thus the Sun faces us with a hierarchy of discrete magnetic elements, ranging from large sunspots (diameter $2 R \cong 55000$ $\mathrm{km}$, flux $\Phi \cong 3 \times 10^{14} \mathrm{~Wb}$, central field intensity $B \cong 0.30 \mathrm{~T}$ ), through dark pores $\left(2 R \cong 3500 \mathrm{~km}, \Phi \cong 2 \times 10^{12} \mathrm{~Wb}, B \cong\right.$ $0.23 \mathrm{~T})$, to small bright elements $(2 R \cong 300$ $\mathrm{km}, \Phi \cong 1 \times 10^{10} \mathrm{~Wb}, B \cong 0.15 \mathrm{~T}$ ). These elements are present for both polarities, and they are distributed over the solar disk in complicated and irregular patterns. Moreover, major changes in the field pattern occur within a few months. Thus the solar magnetic field is utterly different from the smooth dipole-like field of the Earth.

We now discuss the physics of the individual magnetic elements in terms of simple fluxtube models. These models are based on the following constraints (see Fig. $3)$ : The fluxtube is vertical and rooted in the convection zone, which extends from the bottom of the photosphere down to a depth of $2 \times 10^{5} \mathrm{~km}$ ( $\cong 1 / 3$ solar radius) in the solar interior (see Gough page 4). The absence of large mass velocities suggests a static mechanical equilibrium: the vertical component of the pressure gradient is balanced by gravity, and the horizontal component by the Lorentz force. 
As to the thermal balance, the fluxtubes are well insulated from the surrounding convection zone. The mean free path for photons is only a few kilometers in the deep photosphere and it decreases rapidly with increasing depth. The magnetic field is too strong for convective turbulence to penetrate the tubes. Hence it is assumed that there is no heat exchange between the fluxtube and its surroundings, except by radiation.

The darkness of sunspots indicates that the energy flow $F_{\text {i }}$ (per unit area) in the interior is much smaller than the energy flow $F_{\mathrm{e}}$ in the ambient convection zone; this is assumed to be true for thin fluxtubes as well. As a result, the internal temperature $T_{\text {i }}$ is smaller than the ambient temperature $T_{e}$, and the internal gas pressure $P_{\mathrm{i}}$ is smaller than the ambient pressure $P_{\mathrm{e}}$. The magnetic field is contained by the gas pressure excess $P_{\mathrm{e}}-\mathrm{P}_{\mathrm{i}}$; for slender flux tubes this gas pressure excess balances the magnetic pressure $B^{2} /(2 \mu)$.

Magnetostatic models have been computed for thick fluxtubes (sunspots) and for slender fluxtubes (pores and bright elements). With a suitable choice for the internal energy flux $F_{\mathrm{i}}$, that is between $0.1 \mathrm{~F}_{\mathrm{e}}$ and $0.2 F_{e^{\prime}}$ the fluxtube models reproduce the observed properties of the magnetic elements with the observed dependence on size: the spots and the pores are dark because they are large enough that the photosphere in their central parts is sufficiently shielded against radiation from the

Fig. 1 - Large sunspot and pores. The large spot consists of a dark umbra surrounded by a penumbra. Note the penumbral structure of bright and elongated elements that are aligned along the magnetic field. Several pores ("small umbrae without penumbrae") are present at the bottom and to the left. The bright grains outside the spots form the granulation, a convective pattern that covers the entire solar surface. The scale of 10 " corresponds to $7300 \mathrm{~km}$ at the Sun, $1 \%$ of the solar radius (7 July 1970, Observatoire du Pic du Midi, courtesy R. Muller).

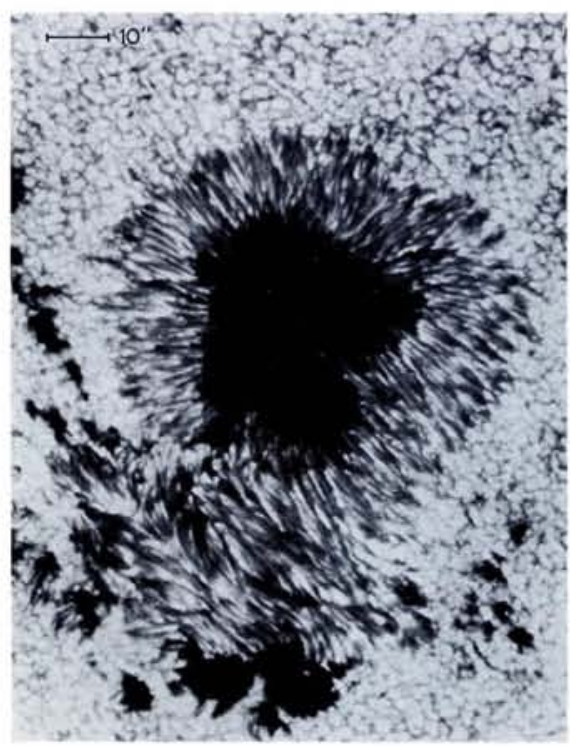

outside. The atmospheric tops of thin fluxtubes, however, are modified by the lateral influx of radiation (Fig. 3), and hence the small magnetic elements show little contrast when observed near the centre of the disk. The magnetic flux density $B$ and the shape of the magnetic field are reproduced with the observed dependence on size.

The temperature reduction $T_{i}<T_{\text {e }}$ extends over several pressure scale heights below the photospheric layer. The result is that the internal gas pressure $P_{i}$ and density $\rho_{\mathrm{i}}$ are small fractions of the external $P_{\mathrm{e}}$ and $\rho_{\mathrm{e}}$ at the same depth. In other words, the isobars and the observed surface (at optical depth unity) are depressed in flux tubes (Fig. 3). In sunspots this "Wilson depression" has been observed since 1769; the apparent position of the umbra within the penumbra moves towards disk centre, as a sunspot approaches the limb. The Wilson depression in thin fluxtubes explains why the small magnetic elements are bright in continuum radiation near the limb: the hot wall surrounding the tube is seen when the tube is obliquely observed.

The low density in the top of the fluxtubes has two more consequences. First, as already noted the fluxtubes are buoyant, which explains their vertical orientation; second, the fluxtube is stabilized against the interchange instability, because the plasma in the fanning fluxtube floats on the heavier plasma outside.

The rapid decrease of the gas pressure with height causes the fan shape of the fluxtube in the atmosphere (Fig. 3). In the convection zone and photosphere the magnetic pressure $B^{2} /(2 \mu)$ is balanced by the excess gas pressure $P_{\mathrm{e}}-P_{\mathrm{i}} \cong P_{\mathrm{e}}$; the Lorentz force ensuring this balance operates in the current sheet in the fluxtube wall. However, within a few pressure scale heights $\left(H_{p} \cong 150 \mathrm{~km}\right)$ above the photosphere, the magnetic field must change to a potential field - similar indeed to the field at the end of a long solenoid. At large heights, the field approaches a monopole structure, the intensity $B$ varying with the inverse square of height if there are no other fluxtubes nearby.

Thus the fraction of the atmosphere occupied by magnetic field increases rapidly with height. Moreover, the ratio between the gas pressure and the magnetic pressure $\beta=2 \mu P / B^{2}$ within magnetic structure, decreases rapidly with height, from $\beta \cong 1$ at the top of the convection zone, to $\beta<<1$ in the outer atmosphere.

The fanning of the fluxtubes and the decrease of $\beta$ with height explain the drastic differences in the appearance of the successive atmospheric layers: photosphere, chromosphere and corona. The deep photosphere is largely free of strong magnetic field, hence the "white-light" face of the Sun is covered with nonmagnetic convective structure (the granulation, see Fig. 1). Magnetic structure
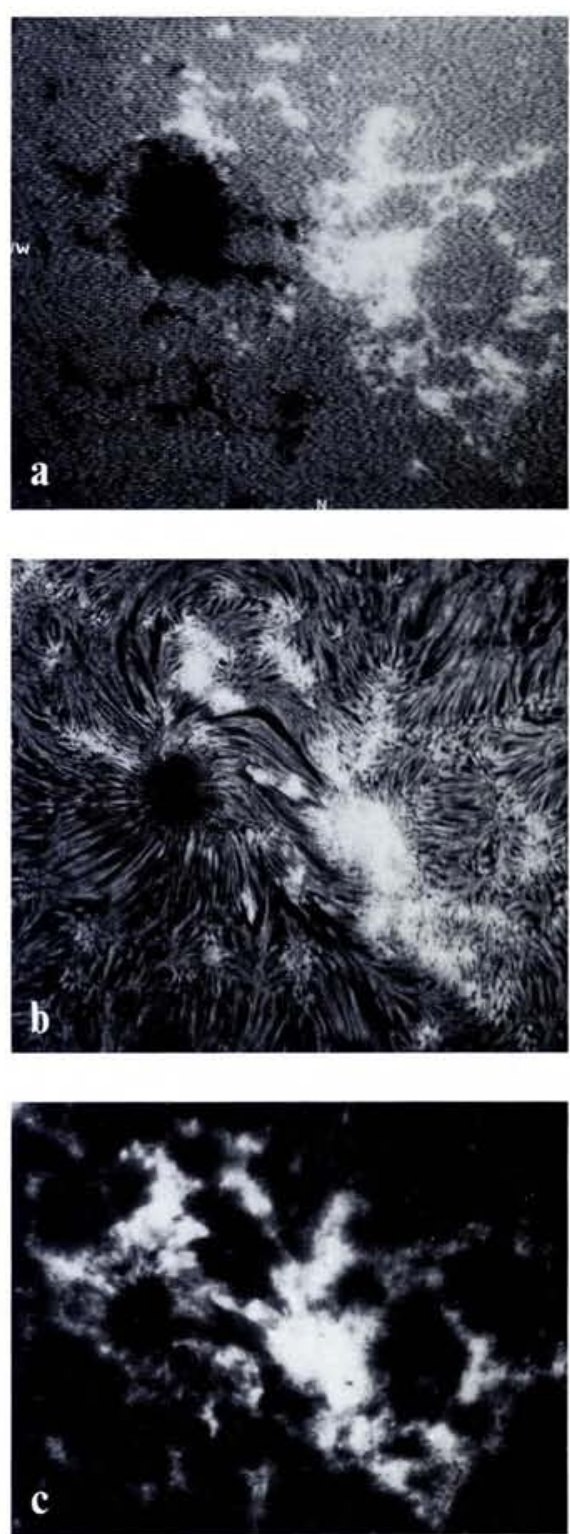

Fig. 2 - An active region: (a) magnetogram, (b) filtergram taken in the core of the Balmer line $\mathrm{H \alpha},(\mathrm{c})$ filtergram taken in the core of the Ca I/ $\mathrm{K}$ resonance line. In the magnetogram, black and white indicate the opposite polarities. Most of the flux of "black" polarity is contained in the large sunspot visible in (b). Small magnetic patches of either polarity are visible as bright elements (faculae) in the $\mathrm{Ca} / \mathrm{K}$ line core; note the close spatial correspondence between the faculae in (c) and the magnetic features in (a). Dense concentrations of faculae are called plages; most of the "white" polarity is concentrated in the large plage to the right of the sunspot. Near the top and the bottom of the frames (a) and (c) is seen the network: patches of magnetic field crudely arranged around empty cells of about $30000 \mathrm{~km}$ diameter. A similar but much weaker network covers the entire solar surface. The filtergram in $\mathrm{H} \alpha$ shows a structure of elongated dark "fibrils" which follow strongly inclined magnetic field lines located at the edges of the areas of strong vertical field shown in (a) 15 July 1973, Big Bear Solar Observatory, courtesy H. Zirin).

(spots, faculae and network) occupies only a tiny fraction of the surface. At larger heights, in the chromosphere, a substantial fraction of the atmosphere is filled with 
strong magnetic field $(\beta<1)$, which explains the intricate structure visible in strong spectral lines (see Fig. 2,b). The corona, the outer atmosphere which can be observed in radio waves and in soft $\mathrm{X}$-rays (see Chiuderi page 8 ), is pervaded by a strong magnetic field (i.e., $\beta<<1$ ); at these heights the fluxtubes have lost their individuality. Instead, the magnetic structure in the corona is determined by the group patterns of magnetic elements in the photosphere.

The magnetic field determines the very existence of the chromosphere and corona by controlling the energy supply. In the top of the convection zone, just below the photosphere, magnetohydrodynamic waves are excited in the fluxtubes. These waves are guided along the fluxtubes into the outer atmosphere, where they dissipate. The observed spatial correlation between magnetic field and emission in the cores of spectral lines present in the small elements (Fig. 2) indicates that the mechanical heating already occurs in the low chromosphere, just above the white-light photosphere (Fig. 3 ).

The magnetic fluxtubes are believed to be generated in the convection zone. The magnetic elements appear in bipolar arrangements called active regions (Fig. 2), with the line connecting the poles nearly parallel to the equator. The emergence and evolution of active regions indicates a horizontal east-west system of flux tubes in the solar interior. An active region is formed when a loop of a bundle of fluxtubes rises into the atmosphere. The compactness of the emerging active regions indicates that the magnetic field in the solar interior is stronger than $0.03 \mathrm{~T}$, which is one of the problems to be solved by the solar dynamo theory (see Schüssler pages 6/8).

The complete hierarchy of magnetic elements is present at the maximum development of a large active region. Sunspots are formed within a week from the first emergence of flux by the coalescence of many small magnetic elements. They start to decay immediately, most of them disappearing in a few weeks. Only the small flux-

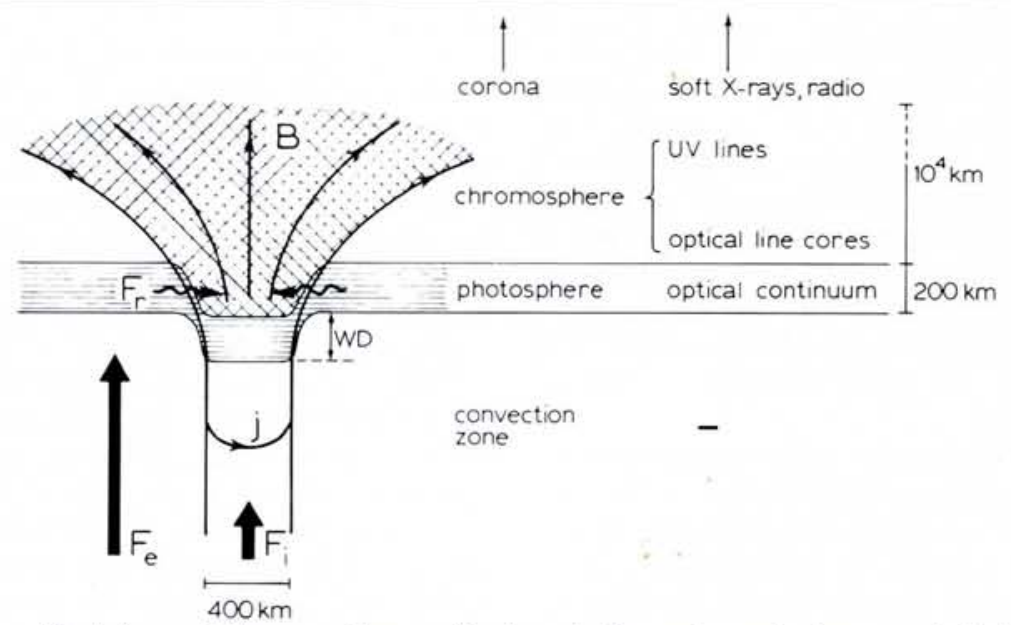

Fig. 3 - Fluxtube model for a small magnetic element. The various solar layers are indicated with their spectral diagnostics; note the Wilson depression (WD). In the photosphere and convection zone the flux tube is bounded by azimuthal currents $j$. The arrows $F_{j}$ and $F_{e}$ indicate energy fluxes per unit area; the wavy arrows $\mathrm{F}_{\mathrm{r}}$ stand for the lateral radiative influx into the transparent top of the fluxtube. The cross-hatching symbolizes the non-thermal heating of the chromosphere.

tubes remain; their clusters (plages) disperse, and after several months the active region is lost in the network, that is the fragmented cellular-structured field covering the Sun. The magnetic flux must disappear again from the atmosphere, but the actual process of disappearance has not yet been observed.

The physics of solar magnetic structure is a key topic in modern astrophysics, one reason being that the magnetic structure reveals the interplay between rotation, convection and magnetic field in the solar interior. Another reason is that magnetic tubes transport mechanical energy from the convection zone into the corona, which heats the corona and so drives the solar wind. The solar wind, streaming out along the magnetic field, determines the loss rate of solar angular momentum.

The topic solar activity has recently been enriched by a stream of stellar data: the application of diagnostics developed in solar physics now reveals that stars with convective envelopes display phenomena similar to solar magnetic activity, the level decreasing with decreasing stellar rotation rate. So it appears that the basic parameters determining stellar structure and evolution, viz. the initial mass and the initial chemical composition of a star, must be complemented by the initial angular momentum. The time-dependent processes of nuclear reactions, which change the stellar thermal structure, must be complemented by the losses of mass and angular momentum, which change the stellar dynamo.

We depend on our nearest star, the Sun, to study the physical processes underlying the magnetic structure and heating of stellar coronae. The Sun permits observation of magnetic activity over its wide range of spatial and temporal scales, with a rich variety of spectroscopic and polarimetric diagnostics. Challenging problems are posed by the processes occurring in the smallest magnetic elements, with diameters at or below the resolution limit occasionally reached with present groundbased instruments. This is one of the reasons why solar physicists strive for the realization of a Large European Solar Telescope, (LEST), with an aperture larger than $2 \mathrm{~m}$, and why they are eagerly looking forward to the Solar Optical Telescope (SOT), to be operated from the Space Shuttle in 1987, and to the Grazing Incidence Solar Telescope.

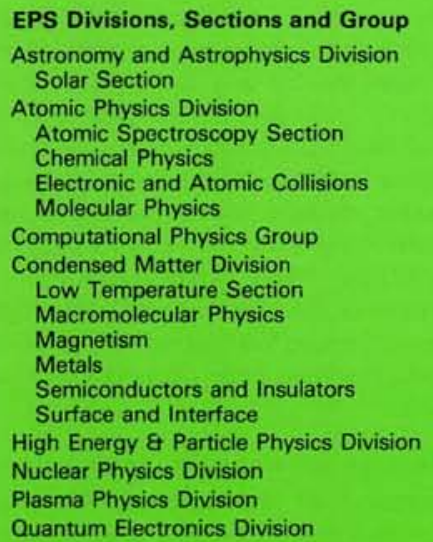

Europhysics News is the official journal of the European Physical Society which comprises 28 National Societies, Academies and Groups, over 3000 Individual Members and 32 Associate Members. Governing bodies of EPS are the General Meeting. Council and an elected Executive Committee responsible for detailed policy. EPS promotes the collaboration of physicists throughout Europe and encourages all aspects of international exchange in physics. EPS administers scholarships awarded for research and studies in different countries and a "teaching abroad" scheme. EPS publishes in addition to EN, Europhysics Conference Abstracts, E. Ed. News and, in collaboration with The Institute of Physics (UK), the European Journal of Physics. Individual Members receive EN free of charge (price to institutions: Sw.Fr, 82. -/a), rebates on the price of many publications and on conference fees. Annual subscription to EPS from Individual Members who belong to one of the EPS member societies is: $\mathrm{Sw} . \mathrm{Fr}$. 36. - ; for independent members: Sw.Fr. 126. - .

\section{Editor: E.N. Shaw}

Meetings Compilation: W.S. Newman

Editorial Board:

K. Appert, A. Baratoff, G.J. Béné,

G.R. Macleod, A. Maeder, J. Muller

Editorial and Advertising Office at the EPS Secretariat.

Address: EUROPEAN PHYSICAL SOCIETY P. O. Box 69 . CH-1213 Petit-Lancy 2 Switzerland

Telephone: Geneva (22) 931130

Telex: 23455 alarm ch

Cables: europhys genève

Printed by: Pfirter frères SA $\mathrm{CH}-1213$ Petit-Lancy/Switzerland 